\title{
Endoscopic Dilation of Corrosive Strictures: Is It Safe?
}

\author{
Bashar Sharma ${ }^{1} \cdot$ John Birk ${ }^{1}$
}

Accepted: 15 October 2021 / Published online: 5 November 2021

(C) The Author(s), under exclusive licence to Springer Science+Business Media, LLC, part of Springer Nature 2021

Ingestion of caustic substances can severely injure the esophagus and stomach, with complications including bleeding, perforation, fistulation, and stricture formation. Though caustic ingestion is usually accidental in children, in adults, it is usually more severe, commonly seen with suicidal attempts [1].

Esophageal strictures are one of the most common longterm complications of caustic ingestion. They can be seen as early as 3 weeks post-ingestion or as late as 1 year [1]. Strictures are also more common following deep circumferential ulcerations and necrosis classified as grades $2 \mathrm{~B}$, $3 \mathrm{~A}$, or $3 \mathrm{~B}$ according to Zargar's endoscopic classification of mucosal injury [2]. They are also more commonly seen with alkali than acid ingestion, since the higher surface tension of alkalis causes liquefactive necrosis, increasing contact time with the esophageal mucosa [3]. In contrast, acids cause coagulative necrosis that forms an eschar that protects the esophagus from further injury [3]. Moreover, larger volumes of alkali are often ingested since acid usually is difficult to ingest due to noxious distaste and oral burning sensation.

Gastric strictures are also seen with severe caustic injuries and can result in gastric outlet obstruction (GOO) due to prepyloric stricturing, due to antral pooling of the caustic agent [1]. Though management of GOO secondary to caustic ingestion is primarily surgical, there are some reports of successful endoscopic dilation $[4,5]$.

Endoscopic dilation is the mainstay of non-surgical treatment of caustic strictures. This can be achieved by bougie dilators such as Savary-Giliard (SG) dilators or by "through the scope" (TTS) balloon dilators. Conventional wisdom dictates that bougies are more reliable and safer than balloons in the management of long fibrotic strictures that

John Birk

birk@uchc.edu

Bashar Sharma

bsharma@uchc.edu

1 Division of Gastroenterology and Hepatology, University of Connecticut, Farmington, CT, USA follow caustic ingestion since they produce a more gradual radial force and provide tactile feedback on dilatation [6]. In general, dilations are avoided within the first 3 weeks following ingestion due to the higher risk of perforation during this time, since the tensile strength of the esophagus and stomach is the lowest given that collagen deposition occurs $\geq 15$ days after caustic injury [2]. The interval between dilation sessions is usually 3-4 weeks. The reported risk of perforation after endoscopic dilation is higher in caustic strictures compared with other benign esophageal strictures: $0.4-32 \%$ versus $0.1-0.4 \%$, respectively [6].

In this issue of Digestive Diseases and Sciences, Bush et al. [7], present a systematic review and a meta-analysis evaluating the risk of perforation from endoscopic dilation of corrosive strictures in adults. They included 15 studies (8 retrospective and 7 prospective) that included 712 patients who underwent 4840 dilations. Their primary outcome was the pooled cumulative perforation rate after dilation, expressed as a percentage of the total study population and the total number of dilation sessions. The secondary outcomes included the perforation rate with balloon vs SG, the type of management required for these perforations (conservative vs surgery), and the mortality rate. They found that the pooled prevalence of perforations as a percentage of the total subjects was $9 \%$, whereas the pooled prevalence of perforations as a percentage of the total number of dilation sessions was $1 \%$, likely reflecting the need for multiple dilation sessions for each patient. The overall mortality was $4.7 \%$, certainly high but reasonable considering the severity of the injury. These data support a relatively low per procedure risk that should reassure endoscopists seeking to dilate these strictures.

Currently, the standard of care in the management of most caustic ingestion strictures is endoscopic treatment. The significance of this study is that it supplies important guidance on an intervention that is performed only occasionally by endoscopists. There has been much heterogeneity in the previous published studies about this topic which is likely secondary to differences in sample size, the types of dilators used, and the endoscopists' experience over the years. 
Having such a large systematic review and meta-analysis is of great importance in order to help clarify some of these differences.

The authors effectively discuss their results explaining some of the factors that could have contributed to the lower perforation rates observed in their study compared with prior publications. Sample size is a major factor; this study included a large sample as discussed above. Moreover, out of the 15 studies included in the review, 12 were published after the year 2000, likely reflecting improvements in patient selection and endoscopic technique over the years combined with improvement over time in the design and physical properties of the types of dilators used.

The study found no difference in the risk of perforation between SG and balloon dilators, consistent with some of the recent reviews that compared both modalities in the management of benign esophageal stricture, showing no difference in regards to symptomatic relief, recurrence, bleeding, and perforation with the exception of less severe post-procedural pain with balloon dilation [8]. These latter findings are notable for their discordance with conventional wisdom.

None of the studies included in the review followed a randomized controlled design. Besides, they all had moderate-high risk of bias which likely affected the results and their generalizability. Furthermore, studies with both esophageal and gastric strictures were included in the review, which further diluted the conclusions, since dilation of esophageal strictures is quite different than gastric dilation, with different outcomes and risks. Lastly, data regarding the starting and maximum diameters of the dilators, as well as the diameter at which the perforations occurred, were not reported in the studies.

In conclusion, the authors should be commended for addressing a topic of great importance to the pediatric and adult populations alike, since foregut strictures are commonly encountered during clinical practice. Further randomized controlled trials comparing both dilators headto-head stratified for esophageal and gastric strictures are needed in order to answer some of the questions that were raised in this review.

\section{Declarations}

Conflict of interest The authors have no disclosures.

\section{References}

1. Contini S, Scarpignato C. Caustic injury of the upper gastrointestinal tract: a comprehensive review. World J Gastroenterol. 2013;19:3918-3930. https://doi.org/10.3748/wjg.v19.i25.3918.

2. Zargar SA, Kochhar R, Mehta S, Mehta SK. The role of fiberoptic endoscopy in the management of corrosive ingestion and modified endoscopic classification of burns. Gastrointest Endosc. 1991;37:165-169. https://doi.org/10.1016/s0016-5107(91) 70678-0.

3. De Lusong MAA, Timbol ABG, Tuazon DJS. Management of esophageal caustic injury. World J Gastrointest Pharmacol Ther. 2017;8:90-98. https://doi.org/10.4292/wjgpt.v8.i2.90.

4. Kochhar R, Malik S, Gupta P et al. Etiological spectrum and response to endoscopic balloon dilation in patients with benign gastric outlet obstruction. Gastrointest Endosc. 2018;88:899-908. https://doi.org/10.1016/j.gie.2018.06.037.

5. Kochhar R, Dutta U, Sethy PK et al. Endoscopic balloon dilation in caustic-induced chronic gastric outlet obstruction. Gastrointest Endosc. 2009;69:800-805. https://doi.org/10.1016/j.gie.2008.05. 056.

6. Arunachalam R, Rammohan A. Corrosive Injury of the upper gastrointestinal tract: a review. Arch Clin Gastroenterol 2016;2:056062. https://doi.org/10.17352/2455-2283.000022.

7. Bush N, Bhattacharjee S, Sachan A et al. Perforations from endoscopic dilation of corrosive strictures in adults: a systematic review and meta-analysis. Dig Dis Sci. (Epub ahead of print). https://doi.org/10.1007/s10620-021-07295-6.

8. Josino I, Madruga-Neto A, Ribeiro I, et al. Endoscopic dilation with bougies versus balloon dilation in esophageal benign strictures: systematic review and meta-analysis. Gastroenterol Res Pract. 2018;2018:5874870. https://doi.org/10.1155/2018/58748 70.

Publisher's Note Springer Nature remains neutral with regard to jurisdictional claims in published maps and institutional affiliations. 